\title{
MÉTODO TITRIMÉTRICO PARA DETERMINAR FOSFITO EM AMOSTRAS AGROINDUSTRIAIS
}

\author{
Vanessa Pezza Franzini e José Anchieta Gomes Neto* \\ Departamento de Química Analítica, Instituto de Química, Universidade Estadual Paulista, CP 355, 14801-970 Araraquara - SP, Brasil
}

Recebido em 4/11/05; aceito em 14/6/06; publicado na web em 10/1/07

\begin{abstract}
TITRIMETRIC METHOD FOR PHOSPHITE DETERMINATION IN AGRICULTURAL CHEMICAL SAMPLES. A new titrimetric method for the determination of phosphite in fertilizer samples, based on reaction of $\mathrm{H}_{3} \mathrm{PO}_{3}$ with standard iodine solution in neutral media, is proposed. Diluted samples containing ca. $0.4 \% \mathrm{~m} / \mathrm{v} \mathrm{P}_{2} \mathrm{O}_{5}$ are heated and titrated with $0.05 \mathrm{~mol} \mathrm{~L}^{-1}$ iodine standard until the solution becomes faint yellow. Back titration is also feasible: a slight excess of titrant is added followed by starch indicator and titration is completed taking as the end point the change in color from blue to colorless. The influence of chemical composition and $\mathrm{pH}$ of buffers, temperature and foreign species on waiting time and end-point detection were investigated. For the $\mathrm{Na}_{2} \mathrm{HPO}_{4} /$ $\mathrm{NaH}_{2} \mathrm{PO}_{4}$ buffer $(\mathrm{pH} 6.8)$ at $70{ }^{\circ} \mathrm{C}$, the titration time was $10 \mathrm{~min}$, corresponding to about $127 \mathrm{mg}$ iodine, $200 \mathrm{mg} \mathrm{KI}$ and $174 \mathrm{mg}$ $\mathrm{Na}_{2} \mathrm{HPO}_{4}$ and $176 \mathrm{mg} \mathrm{NaH}_{2} \mathrm{PO}_{4}$ consumed per determination. Accuracy was checked for phosphite determination in seven fertilizer samples. Results obtained by the proposed procedure were in agreement with those obtained by spectrophotometry at $95 \%$ confidence level. The R.S.D. $(n=10)$ for direct and back titration was $0.4 \%$ and $1.3 \%$ respectively.
\end{abstract}

Keywords: phosphite; titrimetry; iodine.

\section{INTRODUÇÃO}

O uso recente de produtos à base de fosfito nas atividades agrícolas brasileiras tem crescido significativamente em função da busca por aumento na produtividade e na qualidade dos produtos finais ${ }^{1-3}$. Entre as principais vantagens da utilização de fosfito na agricultura merecem destaque a absorção mais rápida de fósforo pela planta em comparação com produtos à base de fosfato, o baixo custo relativo da matéria-prima, o prolongamento do tempo de conservação do produto após a colheita, e por fim, a ação dupla do fosfito, ou seja, além de fertilizante, ele atua como fungicida ${ }^{2,4}$. Nesse contexto, o desenvolvimento e a aplicação de novos métodos analíticos para determinação de fosfito em insumos agroindustriais são importantes não apenas do ponto de vista acadêmico, mas também do social e econômico: dispondo-se de um controle de qualidade eficiente, há grandes chances de se gerar mais confiança em toda a cadeia produtiva e consumidora.

A espectrofotometria e a gravimetria são as técnicas analíticas mais utilizadas para determinação de fósforo ${ }^{5,6}$. Dos métodos espectrofotométricos, destacam-se o do Azul de Molibdênio e o do Amarelo de Molibdênio ${ }^{7}$. Para aplicá-los na determinação qualitativa ou quantitativa de fosfito, o pré-requisito essencial é a conversão prévia dos íons $\mathrm{PO}_{3}{ }^{3-}$ em $\mathrm{PO}_{4}^{3-}$, para que ocorram as respectivas reações indicadoras principais.

Em relação à gravimetria, um dos métodos descritos na literatura $^{6}$ baseia-se na precipitação de fosfato sob a forma de $\mathrm{Mg}\left(\mathrm{NH}_{4}\right) \mathrm{PO}_{4} 6 \mathrm{H}_{2} \mathrm{O}$ ou de pirofosfato de magnésio $-\mathrm{Mg}_{2} \mathrm{P}_{2} \mathrm{O}_{7}$. Devese salientar que a precipitação de fosfato como sal duplo de magnésio e amônio exige também a conversão prévia de fosfito a fosfato empregando-se oxidantes fortes e fontes de aquecimento por períodos relativamente longos. Outro método gravimétrico sugerido na literatura ${ }^{8}$ é aquele baseado na formação de $\mathrm{Hg}_{2} \mathrm{Cl}_{2(\mathrm{~s})}$, conforme equação química representando a reação de óxi-redução e precipitação:

$2 \mathrm{HgCl}_{2(\mathrm{aq})}+\mathrm{H}_{3} \mathrm{PO}_{3(\mathrm{aq})} \rightarrow \mathrm{Hg}_{2} \mathrm{Cl}_{2(\mathrm{~s})}+\mathrm{H}_{3} \mathrm{PO}_{4(\mathrm{aq})}+2 \mathrm{HCl}_{\text {(aq) }}$

*e-mail: anchieta@iq.unesp.br
A vantagem deste último método sobre o primeiro reside na possibilidade de análise direta da amostra. Por outro lado, esse método apresenta a desvantagem de produzir quantidades relativamente grandes de resíduos à base de mercúrio. Isso contraria um dos princípios da Química Verde, segundo o qual se deve evitar o uso de reagentes de difícil descarte no ambiente ${ }^{9}$.

Levando-se em consideração que os métodos disponíveis para fosfito apresentam restrições de caráter ambiental (resíduos de mercúrio) ou alguma periculosidade (longos períodos de exposição do analista a reagentes perigosos, em função do preparo da amostra) e que há demanda por controle de qualidade de um insumo importante do setor agrícola nacional, pois há carência de método simples e rápido para fosfito, o desenvolvimento de um método robusto de análise qualitativa e quantitativa para fosfito é de relevância e tem grandes chances de fornecer resultados positivos, tanto do ponto de vista acadêmico como do setor produtivo. Assim, o principal objetivo deste trabalho foi o desenvolvimento de um método titrimétrico para, respectivamente, padronizar reagentes e determinar fosfito em amostras agroindustriais.

\section{PARTE EXPERIMENTAL}

\section{Materiais}

\section{Instrumentação}

Foi utilizada vidraria de grau A e as pesagens foram feitas em balança analítica Sartorius BL 210 S. O sistema titrimétrico empregou bureta de grau A e agitador magnético com controle de temperatura Marconi MA 085.

\section{Reagentes, soluções e amostras}

Todas as soluções foram preparadas com água purificada pelos sistemas de osmose reversa Rios 5 e desionizador Milli-Q academic, ambos Millipore. Todos os reagentes empregados no trabalho foram de pureza analítica.

Solução $0,05 \mathrm{~mol} \mathrm{~L}^{-1} \mathrm{I}_{2}$ : dissolveu-se $10 \mathrm{~g} \mathrm{KI}$ (Mallinckrodt) em $20 \mathrm{~mL}$ de água em balão de $500 \mathrm{~mL}$; adicionou-se 6,34 $\mathrm{g} \mathrm{I}_{2}$ sublimado (Spectrum), agitou-se até dissolução do iodo e, em seguida, 
completou-se o volume com água. A solução final foi padronizada com solução $0,05 \mathrm{~mol} \mathrm{~L}^{-1} \mathrm{Na}_{2} \mathrm{~S}_{2} \mathrm{O}_{3}$ e armazenada em frasco de vidro âmbar.

Solução $0,05 \mathrm{~mol} \mathrm{~L}^{-1} \mathrm{Na}_{2} \mathrm{~S}_{2} \mathrm{O}_{3}$ : dissolveu-se $6,20 \mathrm{~g} \mathrm{Na}_{2} \mathrm{~S}_{2} \mathrm{O}_{3} \cdot 5 \mathrm{H}_{2} \mathrm{O}$ (Spectrum) em cerca de $200 \mathrm{~mL}$ de água, transferiu-se a solução para balão de $500 \mathrm{~mL}$ e completou-se o volume com água. Essa solução foi padronizada com solução $0,0067 \mathrm{~mol} \mathrm{~L}^{-1} \mathrm{~K}_{2} \mathrm{Cr}_{2} \mathrm{O}_{7}$.

Solução $0,0067 \mathrm{~mol} \mathrm{~L}^{-1} \mathrm{~K}_{2} \mathrm{Cr}_{2} \mathrm{O}_{7}$ : dissolveu-se em água $0,49 \mathrm{~g}$ $\mathrm{K}_{2} \mathrm{Cr}_{2} \mathrm{O}_{7}$ (Mallinckrodt) seco a $140{ }^{\circ} \mathrm{C}$ por $2 \mathrm{~h}$, transferiu-se a solução para balão volumétrico de $250 \mathrm{~mL}$ e completou-se o volume com água.

Suspensão indicadora de amido: uma pasta contendo $1 \mathrm{~g}$ de amido foi dispersa em $100 \mathrm{~mL}$ de água fervente e, em seguida, foram adicionados $2 \mathrm{~g}$ KI, agitou-se para dissolução e homogeneização e armazenou-se a solução final em frasco âmbar.

Solução $0,05 \mathrm{~mol} \mathrm{~L} \mathrm{Na}_{2} \mathrm{HPO}_{3} \cdot 5 \mathrm{H}_{2} \mathrm{O}$ : pesou-se $5,4 \mathrm{~g}$ do sal (Riedel-de Haën) e dissolveu-se em água, transferiu-se a solução para balão volumétrico de $500 \mathrm{~mL}$ e completou-se o volume com água. Esta solução foi padronizada via iodimetria.

Diferentes soluções-tampão no intervalo de $\mathrm{pH}$ de 3,0 a 7,8 foram preparadas a partir de misturas apropriadas de soluções 0,2 mol L ${ }^{-1}$ de mono-hidrogênio fosfato de sódio, $0,2 \mathrm{~mol} \mathrm{~L}^{-1}$ de dihidrogênio fosfato de sódio, $0,2 \mathrm{~mol} \mathrm{~L}^{-1}$ ácido acético, $0,2 \mathrm{~mol} \mathrm{~L}-1$ acetato de sódio ou $0,1 \mathrm{~mol} \mathrm{~L}^{-1}$ ácido cítrico ${ }^{10}$.

As amostras de fertilizantes foram fornecidas pela Indústria e Comércio Samaritá Ltda (Artur Nogueira - SP). As amostras foram pesadas e diluídas 1:50 ou 1:100 (m/v) em água.

\section{Método}

A influência do pH e da temperatura no tempo de obtenção do ponto final foram avaliadas titulando-se $10,00 \mathrm{~mL}$ de solução 0,05 mol L-1 $\mathrm{Na}_{2} \mathrm{HPO}_{3} .5 \mathrm{H}_{2} \mathrm{O}$ diluídos em $25,00 \mathrm{~mL}$ de diferentes soluções tampão $(3,0 ; 4,0 ; 5,0 ; 5,8 ; 6,0 ; 6,8 ; 7,0$ e 7,8) com solução $0,05 \mathrm{~mol} \mathrm{~L}^{-1} \mathrm{I}_{2}$ a duas temperaturas, 25 e $70{ }^{\circ} \mathrm{C}$. O titulante foi adicionado até que a cor amarelo pálido tivesse sido observada, indicando assim o ponto final da titulação. Para a titulação de retorno o seguinte procedimento foi adotado: o frasco de titulação foi retirado do aquecimento, foram adicionados $2,00 \mathrm{~mL}$ de solução $0,05 \mathrm{~mol} \mathrm{~L}^{-1} \mathrm{I}_{2}$ em excesso, o frasco foi tampado e mantido ao abrigo da luz até que a solução atingisse a temperatura ambiente. Em seguida, o excesso de iodo foi titulado com solução $0,05 \mathrm{~mol}$ $\mathrm{L}^{-1} \mathrm{Na}_{2} \mathrm{~S}_{2} \mathrm{O}_{3}$, utilizando solução de amido como indicador.

A influência das principais espécies químicas normalmente presentes em fertilizantes líquidos foi avaliada titulando-se $10,00 \mathrm{~mL}$ de solução $0,05 \mathrm{~mol} \mathrm{~L}^{-1} \mathrm{Na}_{2} \mathrm{HPO}_{3} .5 \mathrm{H}_{2} \mathrm{O}$ diluídos em $25,00 \mathrm{~mL}$ de

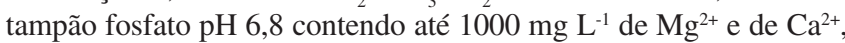
$200 \mathrm{mg} \mathrm{L}^{-1}$ de $\mathrm{Zn}^{2+}$ e de $\mathrm{Mn}^{2+}, 30 \mathrm{mg} \mathrm{L}^{-1}$ de $\mathrm{Cu}^{2+}, 10.000 \mathrm{mg} \mathrm{L}^{-1}$ de $\mathrm{Cl}^{-}, \mathrm{SO}_{4}^{2-}, \mathrm{NO}_{3}^{-}$e $\mathrm{CO}_{3}^{2-}$, e $20 \mathrm{mg} \mathrm{L}^{-1}$ de $\mathrm{COT}$ (ácido húmico). A solução final foi titulada com solução $0,05 \mathrm{~mol} \mathrm{~L}^{-1} \mathrm{I}_{2}$.

Os resultados foram analisados após a aplicação de testes estatísticos bem estabelecidos, com ênfase na precisão e na exatidão, estabelecendo-se os intervalos de confiança da média. A precisão das medidas foi estimada por meio do cálculo do desvio padrão após 10 titulações sucessivas de $10,00 \mathrm{~mL}$ de solução $0,05 \mathrm{~mol} \mathrm{~L}^{-1} \mathrm{Na}_{2} \mathrm{HPO}_{3} .5 \mathrm{H}_{2} \mathrm{O}+25,00$ $\mathrm{mL}$ de tampão $\mathrm{Na}_{2} \mathrm{HPO}_{4} / \mathrm{NaH}_{2} \mathrm{PO}_{4}(\mathrm{pH}=6,8)$ e temperatura controlada $\left(70 \pm 1{ }^{\circ} \mathrm{C}\right)$. A avaliação da existência de uma diferença significativa na exatidão e na precisão entre o conjunto de dados obtido pelo método proposto e o conjunto obtido pelo método espectrofotométrico em fluxo $^{11}$ foi feita pelos testes $t$ e $F$, respectivamente ${ }^{12}$.

\section{RESULTADOS E DISCUSSÃO}

Há vários métodos que utilizam iodo como oxidante na determina- ção de diferentes analitos como As, $\mathrm{Sb}, \mathrm{Sn}, \mathrm{H}_{2} \mathrm{~S}, \mathrm{SO}_{2}, \mathrm{~S}_{2} \mathrm{O}_{3}{ }^{2-}, \mathrm{N}_{2} \mathrm{H}_{4}$ e ácido ascórbico ${ }^{13}$. Contudo, nenhuma aplicação para fosfito foi encontrada. Testes preliminares ao estudo proposto neste artigo confirmaram o potencial da iodimetria na determinação de fosfito. O princípio do método baseia-se na oxidação de fosfito a fosfato segundo a reação:

$$
\mathrm{H}_{3} \mathrm{PO}_{3}+\mathrm{I}_{2}+\mathrm{H}_{2} \mathrm{O} \rightarrow \mathrm{H}_{3} \mathrm{PO}_{4}+2 \mathrm{H}^{+}+2 \mathrm{I}^{-}
$$

Na titulação direta, o ponto final é identificado pelo aparecimento da cor amarelo pálido no meio reacional, em função do excesso de iodo. Alternativamente, a titulação por retorno também é possível titulando-se o excesso do oxidante com solução de $\mathrm{Na}_{2} \mathrm{~S}_{2} \mathrm{O}_{3}$ utilizando solução de amido como indicador ${ }^{8}$.

Os primeiros experimentos foram conduzidos à temperatura ambiente $\left(c a .25^{\circ} \mathrm{C}\right)$ e revelaram uma cinética reacional lenta, pois as primeiras gotas do titulante adicionadas já imprimiam ao titulado a coloração do ponto final. Aumentando-se a temperatura para $60{ }^{\circ} \mathrm{C}$ a velocidade da reação aumentou expressivamente, uma vez que o iodo adicionado era consumido quase que instantaneamente. Assim sendo, foi feito um estudo da influência da temperatura na reação indicadora. Ainda, como em $\mathrm{pH}<8$ o potencial de redução do par iodo/iodeto é independente do $\mathrm{pH}$, mas em meio mais alcalino o iodo reage com íons hidroxila formando íons hipoiodito e iodeto ${ }^{8}$, o estudo da influência do $\mathrm{pH}$ na reação entre iodo e fosfito mostrou-se igualmente importante. Fixou-se então $60{ }^{\circ} \mathrm{C}$ para os experimentos seguintes envolvendo a avaliação da influência da natureza e do pH da solução tampão. Nesse estudo, uma alíquota de $10,00 \mathrm{~mL}$ de solução $0,05 \mathrm{~mol} \mathrm{~L}^{-1}$ $\mathrm{Na}_{2} \mathrm{HPO}_{3} .5 \mathrm{H}_{2} \mathrm{O}$ era transferida para a cela de titulação, seguida de $25,0 \mathrm{~mL}$ de solução tampão. A solução de iodo $0,05 \mathrm{~mol} \mathrm{~L}^{-1}$ era adicionada a essa mistura até o estabelecimento do ponto final. Os sistemas tampão avaliados foram: a) ácido acético-acetato de sódio, $\mathrm{pH}$ 4,0 e 5,0; b) $\mathrm{Na}_{2} \mathrm{HPO}_{4}-\mathrm{NaH}_{2} \mathrm{PO}_{4}$, pH 5,8; 6,8; 7,8; c) ácido cítrico-dihidrogenofosfato de sódio, $\mathrm{pH} 3,0 ; 4,0 ; 5,0 ; 6,0 ; 7,0$.

Independentemente da natureza do tampão, a reação entre iodo e fosfito apresentou-se muito lenta para valores de $\mathrm{pH} \leq 4,0$. A titulação nem mesmo foi conduzida até o final: até aproximadamente a metade do ponto de equivalência, a coloração do iodo permanecia por cerca de $20 \mathrm{~min}$. A reação foi ligeiramente acelerada quando o $\mathrm{pH}$ foi aumentado para 5, contudo, o tempo total da titulação (direta mais retorno) foi de aproximadamente $40 \mathrm{~min}$. Um aumento expressivo da velocidade da reação foi observado para os sistemas tampão fosfato e ácido cítrico/fosfato, apresentando $\mathrm{pH}$ entre 5 e 8, uma vez que o iodo adicionado era prontamente consumido. Também a duração da titulação direta foi de 25, 12 e 10 min para o tampão fosfato $\mathrm{pH}=5,8,6,8$ e 7,8, respectivamente. Para ácido cítrico/fosfato, o tempo de titulação foi de 30, 20 e 15 min para pH 5, 6 e 7, respectivamente. Deve ser comentado que o tempo total gasto na titulação de retorno, incluindo o resfriamento da solução, foi de aproximadamente 7 min. Apesar de o tampão fosfato $\mathrm{pH}$ 7,8 ter apresentado o menor tempo de titulação, optou-se por trabalhar com o mesmo sistema tampão, mas a pH 6,8. Apesar do acréscimo de 2 min na titulação nessa condição, a probabilidade de o iodo reagir com os íons $\mathrm{OH}^{-}$ para formar iodeto e hipoiodito ${ }^{8}$ é menor. Determinar fosfito em presença de altas concentrações de fosfatos é interessante porque permite quantificar na amostra o teor de fósforo (expresso como $\mathrm{P}_{2} \mathrm{O}_{5}$ ) proveniente apenas do fosfito. Pelos métodos convencionais de determinação de fosfito, há a necessidade de duas determinações, uma antes e outra após a conversão do analito a fosfato ${ }^{7}$. Foi feito um planejamento experimental quimiométrico para checar esses resultados. A influência do $\mathrm{pH}$ e da temperatura também foi estudada a partir de um planejamento fatorial completo para essas 2 variáveis, nos níveis baixo (-1) e alto (+1). As variáveis experimentais estudadas e a matriz de planejamento experimental estão apresentadas na 
Tabela 1. Esta matriz foi gerada e seus resultados analisados pelo uso do software MINITAB versão 13. A análise das Figuras 1 e 2 revela que as duas variáveis investigadas, assim como a interação entre elas, influenciam significativamente no tempo de reação (resposta). A influência mais significativa é do $\mathrm{pH}$, seguida da temperatura e da interação entre elas. A influência negativa significa que quanto maior o $\mathrm{pH}$ e a temperatura, menor é o tempo de reação. Assim, as melhores condições de $\mathrm{pH}$ e temperatura foram obtidas com tampão $\mathrm{Na}_{2} \mathrm{HPO}_{4} / \mathrm{NaH}_{2} \mathrm{PO}_{4}\left(\mathrm{pH} 6,8\right.$ - 7,0) e $70{ }^{\circ} \mathrm{C}$, respectivamente, e foram as selecionadas para a continuidade dos experimentos. A otimização do método permitiu padronizar o sal $\mathrm{Na}_{2} \mathrm{HPO}_{3} .5 \mathrm{H}_{2} \mathrm{O}$. Esse método titrimétrico para padronização de fosfito é fundamental quando se deseja conhecer com precisão a concentração das soluções de fosfito empregadas em métodos que usam curvas de calibração.

Tabela 1. Matriz do planejamento fatorial da influência do $\mathrm{pH}$ e da temperatura

\begin{tabular}{lcccc}
\hline Ensaios & \multicolumn{2}{c}{ Variáveis não codificadas } & \multicolumn{2}{c}{ Variáveis codificadas } \\
& $\mathbf{p H}$ & $\mathbf{T}\left({ }^{\circ} \mathbf{C}\right)$ & Fator $\mathbf{A}$ & Fator B \\
\hline $\mathbf{1}$ & 3,0 & 25 & -1 & -1 \\
$\mathbf{2}$ & 7,0 & 25 & +1 & -1 \\
$\mathbf{3}$ & 3,0 & 70 & -1 & +1 \\
$\mathbf{4}$ & 7,0 & 70 & +1 & +1 \\
\hline
\end{tabular}

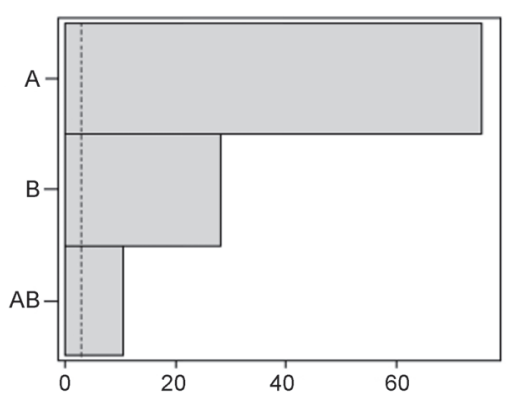

Figura 1. Gráfico de Pareto dos efeitos das variáveis investigadas para $95 \%$ de confiança. $A=p H ; B=$ temperatura; $A B=$ interação entre $p H$ e temperatura

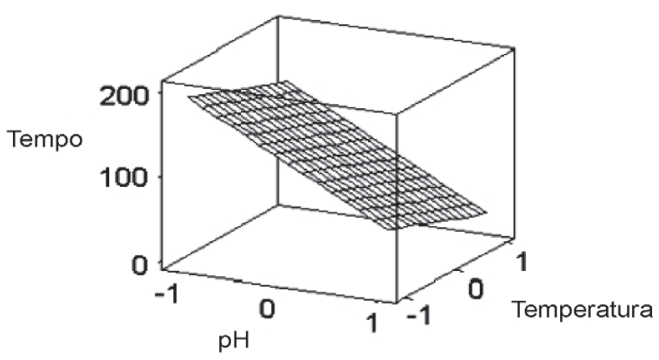

Figura 2. Superfície de resposta correspondente à influência do $\mathrm{pH}$ e da temperatura no tempo de reação

Com objetivo de se estudar a aplicação do método a amostras reais, foi feita uma avaliação da influência de cátions, ânions e matéria orgânica que podem fazer parte de formulações de fertilizantes líquidos à base de fosfito e causar interferências. A concentração das espécies químicas selecionadas foi escolhida em função dos teores de macro e micro nutrientes usualmente presentes nas formulações comerciais. Com exceção do $\mathrm{Mn}$, não se observou interferência dos cátions selecionados. No caso do $\mathrm{Mn}^{2+}$, foi notada interferência positiva de aproximadamente 8,8\% a partir da concentração de $150 \mathrm{mg} \mathrm{L}^{-1}$.

Em relação aos ânions $\mathrm{Cl}^{-}, \mathrm{SO}_{4}^{2-}, \mathrm{NO}_{3}^{-}, \mathrm{CO}_{3}^{2-}$, testados sozi- nhos ou em mistura em concentração de $10.000 \mathrm{mg} \mathrm{L}^{-1}$, foi detectada interferência positiva apenas para $\mathrm{CO}_{3}{ }^{2-}$. Essa interferência foi atribuída ao aumento do $\mathrm{pH}$ do meio da região neutra para a básica, promovendo o consumo de iodo pela reação com íons hidroxila, com a consequiente formação de iodeto e hipoiodito ${ }^{8}$.

A matéria orgânica foi avaliada por meio da adição de ácido húmico de modo a produzir, no titulado, uma solução com concentração de carbono orgânico total (COT) de no máximo $50 \mathrm{mg} \mathrm{L}^{-1}$. Não foi notada qualquer interferência para COT até $20 \mathrm{mg} \mathrm{L}^{-1}$, mas concentrações superiores dificultaram a visualização do ponto final porque a coloração do titulado era semelhante à da solução de iodo. Apesar de a adição intencional de substância húmica (SH) em fertilizantes líquidos contendo fosfito ser uma prática incomum, a finalidade desse estudo foi prever o efeito de tal adição e, também, estabelecer limites quanto à qualidade das águas empregadas na fabricação de tais insumos agrícolas.

A reprodutibilidade do método foi avaliada por meio do desvio padrão relativo de 10 titulações sucessivas. O R.S.D. calculado para a titulação direta e de retorno foi de 0,4 e $1,3 \%$, respectivamente. O desempenho do método titrimétrico proposto foi avaliado após análise de 7 amostras comerciais de fertilizantes líquidos pelo método proposto e por espectrofotometria em fluxo ${ }^{11}$ empregando oxidação em linha e formação do complexo Azul de Molibdênio. Os resultados obtidos estão apresentados na Tabela 2. Aplicando-se o teste $t$ conclui-se que não há diferença significativa entre as médias dos resultados dos dois métodos em nível de confiança de $95 \%$. Para um tempo médio de titulação direta de $10 \mathrm{~min}$, o consumo médio de reagentes corresponde a $200 \mathrm{mg} \mathrm{KI}, 127$ $m g \mathrm{I}_{2}, 174 \mathrm{mg} \mathrm{Na}_{2} \mathrm{HPO}_{4}$ e $176 \mathrm{mg} \mathrm{NaH} \mathrm{PO}_{4}$ por determinação.

Tabela 2. Resultados comparativos de determinação de fosfito [\%(m/ m) $\mathrm{P}_{2} \mathrm{O}_{5}$ ] em fertilizantes líquidos pelo método proposto (titrimetria) e por espectrofotometria ${ }^{11}$

\begin{tabular}{lcc}
\hline Amostra $(\mathrm{N}-\mathrm{P}-\mathrm{K}) *$ & Titrimetria & Espectrofotometria \\
\hline $00-20-20$ & $19,5 \pm 0,1$ & $20,2 \pm 1,0$ \\
$00-28-26$ & $28,3 \pm 0,2$ & $26,0 \pm 3,2$ \\
$00-30-20$ & $29,7 \pm 0,2$ & $29,0 \pm 0,6$ \\
$00-35-10$ & $35,8 \pm 0,2$ & $37,4 \pm 0,3$ \\
$00-40-20$ & $39,1 \pm 0,2$ & $40,0 \pm 0,6$ \\
$00-30-20$ & $30,8 \pm 0,3$ & $30,6 \pm 0,6$ \\
$00-30-20$ & $30,4 \pm 0,2$ & $30,5 \pm 1,3$
\end{tabular}

* Valores rotulados pelo fabricante e expressos em $\%(\mathrm{~m} / \mathrm{m}) \mathrm{N}-$ $\mathrm{P}_{2} \mathrm{O}_{5}-\mathrm{K}_{2} \mathrm{O}$

\section{CONCLUSÃO}

Diferentemente dos métodos gravimétrico e espectrofotométrico convencionais, o método titrimétrico proposto permite determinar fosfito em fertilizantes sem pré-tratamento da amostra, de modo mais rápido e com menor consumo de reagentes, reduzindo assim os custos analíticos. O método espectrofotométrico em fluxo não requer pré-tratamento da amostra, mas utiliza instrumentação mais cara e complexa. Portanto, o método proposto apresenta-se prático, rápido, de baixo custo relativo, de fácil implantação em unidades de fabricação de fosfitos ou até em pontos de distribuição ou consumo ou no próprio campo. As características favoráveis do método habilitam-no a candidato a método oficial.

\section{AGRADECIMENTOS}

À CAPES pela bolsa de doutorado concedida a V. P. Franzini, à FAPESP pelo auxílio financeiro, e ao CNPq pela bolsa concedida a J. A. Gomes Neto. 


\section{REFERÊNCIAS}

1. http://www.conab.gov.br, acessada em Outubro 2005.

2. Afek. U.; Sztejnberg, A.; Phytopathology 1989, 79, 736

3. Katz, M.; Citrograph. 1996, 5, 81.

4. Malavolta, E.; Elementos de Nutrição Mineral de Plantas, Agronômica Ceres Ltda: São Paulo, 1980, p.130-140.

5. Marczenko, Z.; Separation and spectrophotometric determination of elements, Chichester: Ellis Horwood, 1986, p.447-456.

6. Basset, J.; Denney, R. C.; Jeffery, G. H.; Mendham, J.; Análise inorgânica quantitativa, Guanabara Dois: Rio de Janeiro, 1981, p. 245 - 368

7. Babko, A. K.; Pilipenko, A. T.; Photometric Analysis, General Principles and Working Tools, Mir Publishers, 1971

8. Mendham, J.; Denney, R. C.; Barnes, J. D.; Thomas, M. J. K.; Análise química quantitativa, LTC: Rio de Janeiro, 2002, p. $264-375$

9. Silva, L. M.; Santana, M. H. P.; Boodts, J. F. C.; Quim. Nova 2003, 26, 880

10. Perrin, D. D.; Dempsey, B.; Buffers for $\mathrm{pH}$ and metal ion control, Chapman and Hall: London, 1974, p.175.

11. Gomes Neto, J. A.; Ito, H. A.; Fernandes, K. G.; Moraes, M.; Cardoso, A. A.; Laboratory Robotics and Automation 2000, 12, 286.

12. Caulcutt, R.; Boddy, R.; Statistics for Analytical Chemists, Chapman and Hall: New York, 1983.

13. Skoog, D. A.; West, D. M.; Holler, F. J.; Fundamentals of Analytical Chemistry, Saunders College Publishing: New York, 1992, p.387. 